\title{
INTERAÇÕES ENTRE GRANDES EMPRESAS E STARTUPS: O CASO DO CAPITAL EMPREENDEDOR CORPORATIVO
}

Elias Eduardo Bernardo Da Silva ${ }^{1}$ Henrique M Barros ${ }^{1}$

Luiz Ojima Sakuda ${ }^{1}$

\footnotetext{
${ }^{1}$ Centro Universitário FEI
} 


\section{INTERAÇÕES ENTRE GRANDES EMPRESAS E STARTUPS: O CASO DO CAPITAL EMPREENDEDOR CORPORATIVO}

Resumo: O objetivo deste trabalho foi compreender as motivações que têm levado as grandes empresas a utilizar o Capital Empreendedor Corporativo (CEC) como mecanismo para ampliar seus processos de inovação. A metodologia utilizada foi uma abordagem qualitativa através de estudos de caso de duas empresas que utilizam o sistema em seus processos de inovação. A análise dos casos foi complementada por entrevistas com dois especialistas da área. O trabalho foi direcionado para um comparativo das motivações reportadas em trabalhos internacionais e das empresas brasileiras, com recorte específico para o setor do agronegócio. Foi destacada a intersecção das motivações e os direcionadores do cenário brasileiro que, como resultado, observou-se que os ecossistemas de inovação brasileiro estão em processo de amadurecimento: as iniciativas de CEC no Brasil não são consideradas para apoiar ações de elevados riscos e incertezas nem para ampliação das empresas para atendimentos de mercados globais, aspectos levantados na literatura internacional.

Palavras-chave: inovação aberta; capital empreendedor corporativo; agronegócio; capital de risco.

\section{$1 \quad$ Introdução}

A rápida velocidade das mudanças tecnológicas, a hipercompetitividade dos mercados, a globalização e o surgimento de micromercados têm levado as empresas a adotarem estruturas em busca do aumento de competitividade e de capacidade de criação de valor (BADEN-FULLER; HAEFLIGER, 2013; ROMANS, 2016; TEECE; PISANO; SHUEN, 1997). Esse movimento está baseado, em grande medida, no esforço de inovação (KIM; KIM; FOSS, 2016).

A inovação tem sido abordada recorrentemente nas organizações de variados segmentos e propósitos com o objetivo de identificar a forma pela qual elas podem criar diferenciais interagindo com fontes externas de conhecimento (BATTISTI et al., 2015; CHESBROUGH, 2012; VARRICHIO, 2016). A literatura especializada destaca que, para ampliar as oportunidades de inovação, a forma utilizada pelas empresas tem sido explorar o relacionamento com atores externos por meio da estratégia de inovação aberta, com o objetivo de adquirir conhecimentos, codesenvolver e compartilhar aprendizados e experiências de diversas formas (CHESBROUGH, 2012).

Vários modelos organizacionais têm sido implementados para viabilizar o acesso às bases externas de conhecimento (BATTISTI et al., 2015; LOPES; CARVALHO, 2012; TORRES-FREIRE et al., 2013), dentre os quais esta o modelo de Capital Empreendedor Corporativo (CEC), tradução do inglês Corporate Venture Capital - CVC (ARRUDA et al., 2013; BERNARDES et al., 2013; CHESBROUGH, 2002). As empresas estão utilizando o CEC para redefinir suas práticas de inovação e investimentos, bem como para expandir a forma de ampliar as fronteiras da empresa para inovarem com rapidez e conseguirem maior alinhamento com o mercado (ANOKHIN; PECK; WINCENT, 2016; BASU; PHELPS; 
KOTHA, 2011; BATTISTINI; HACKLIN; BASCHERA, 2013). E o CEC está começando a ser mais explorado por setores que ainda não o utilizavam de forma tão intensa, como o agronegócio. (AGROPENSA, 2014; WALKER et al., 2016).

A literatura sobre CEC no Brasil já retratou as iniciativas de investimentos de multinacionais em startups brasileiras (LUIZA et al., 2018). Essa mesma literatura revelou como as empresas têm promovido o intraempreendedorismo corporativo por meio do CEC (SALUSSE; CAMPOS NETO, 2018), e como o CEC se torna fonte de recursos para as aceleradoras (SANTOS FIGUEIREDO; FIGUEIREDO; BRAGA, 2017). No entanto, as motivações e a organização do CEC ainda não estão bem documentadas, especialmente no contexto do agronegócio. Considerando as particularidades da cadeia vinculada ao agronegócio brasileiro, em que as empresas estão se aproximando do ecossistema empreendedor por meio de parcerias (ALUMNI BRASIL, 2017; RIECHE; FARIA, 2014; SAKUDA; CAMPO, 2017) com vistas à geração de inovações (MUNDO NETO; SALTORATO, 2017; RODRIGUES; SANTOS; KUNIYOSHI, 2013), este artigo busca ampliar o conhecimento sobre o uso do CEC nesse contexto com o propósito de colocar luz sobre como as iniciativas de CEC estão sendo estruturadas, explorando não apenas o que motiva as organizações a buscarem o CEC, mas também como o CEC afeta a capacidade de inovar das organizações.

Para tanto, esta pesquisa conduziu a análise de 2 casos, realizando entrevistas com as empresas do agronegócio brasileiro e com agentes externos envolvidos com o CEC nos últimos cinco anos.

\section{$2 \quad$ Revisão da Literatura}

O ambiente empresarial está em contínua evolução, cada vez mais competitivo e dinâmico (KARVINEN-NIINIKOSKI, 2004). As rápidas mudanças dos ciclos tecnológicos e do processo de interligação econômica e social entre as diversas regiões do mundo proporcionam uma dinâmica que demanda modalidades de produção e oferta de serviços diferentes e inovadoras (LUIZA et al., 2018). Assim, o que se observa são as empresas investindo em inovação para manterem a competitividade ou se tornarem líderes (MOHAMED; SCHWIENBACHER, 2016). Teece (2010) descreve que a inovação é um elo para o crescimento das receitas e para a garantia de lucratividade.

Para ampliar as oportunidades de inovação, as empresas têm investido em ações de colaboração (LOPES; CARVALHO, 2012), explorando o relacionamento com atores externos, descentralizando suas atividades de inovação para ampliarem seus processos de absorção de conhecimento (GASSMANN; ENKEL; CHESBROUGH, 2010; VON KROGH et al., 2012). Tal estratégia, conhecida como estratégia de inovação aberta, consiste no uso das possibilidades de geração de inovação por meio de relacionamentos externos (CHESBROUGH, 2012).

Herstatt (2012) descreve que as empresas podem aumentar a probabilidade de oferecer uma proposta de valor atraente, se forem capazes de conectar com êxito seus processos de inovação com as redes de inovação. Assim, as ações para o processo de inovação podem ocorrer em vários formatos, dependendo do objetivo que a empresa pretende atingir e também do nível de risco e do comprometimento que se pretende (COSTA; PORTO, 2013). Um dos mecanismos utilizados pelas empresas, como fonte de conhecimento externo para 
ampliar a capacidade de inovação, é o Capital Empreendedor Corporativo - CEC (LEE; KIM; JANG, 2015).

O CEC pode ampliar a capacidade de inovação das empresas por meio do aproveitamento e da atualização das competências disponíveis. De fato, há evidências de que o capital empreendedor desempenha papel relevante para o desenvolvimento de novas tecnologias, principalmente em empresas nascentes (HENDERSON, 2009). O CEC permite acesso a processos inovadores e ao compartilhamento de custos com os parceiros externos (VON KROGH et al., 2012). E ele se torna um mecanismo estratégico que pode acelerar o ritmo da inovação, proporcionando oportunidades para parcerias estratégicas e viabilizando o desenvolvimento de tecnologias emergentes (BATTISTINI; HACKLIN; BASCHERA, 2013).

\subsection{Capital Empreendedor Corporativo}

As atividades de CEC cresceram significativamente nos últimos anos (THEES, 2016). Dados da CB Insights (Figura 1) demonstram que, em 2018, os fundos de CEC investiram 53 bilhões de dólares em 2.740 negócios, com aumento, em relação ao ano anterior, de $47 \%$ nos valores investidos e de $32 \%$ no número de novos negócios.

\section{Figura 1 - Evolução recente do mercado global de CEC}

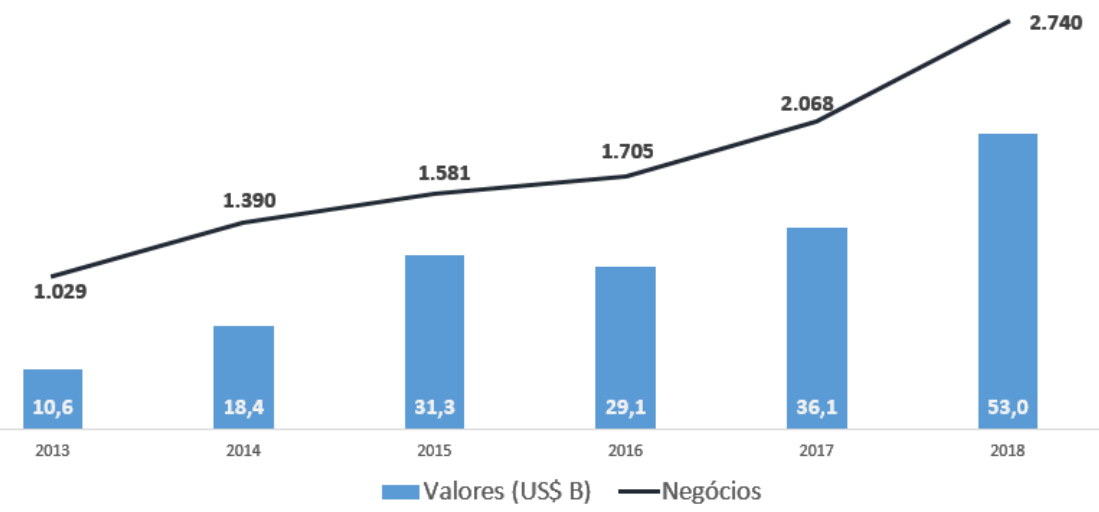

Fonte: CB Insights (2018)

O CEC é uma forma de apoiar o empreendedorismo corporativo, buscando criar organizações, internas ou externas, ou apoiar empresas em início de operação, também conhecidas como startups (CHEMMANUR; LOUTSKINA; TIAN, 2014; CUMMING; FLEMING; SUCHARD, 2005). O CEC é entendido como um conjunto de estratégias e mecanismos usados por empresas maduras para a renovação de sua oferta de produtos e serviços, seja pela criação de novos mercados, pelo lançamento de novos produtos ou pela formação de unidades de negócios autônomas (ARRUDA et al., 2013). Anokhin, Peck e Wincent (2016) acrescentam que o CEC permite explorar o aprendizado das empresas em áreas em que elas não possuem experiência.

A origem do CEC data da década de 1960, quando várias empresas americanas começaram a experimentar fundos corporativos como complemento às atividades internas de desenvolvimento de novos negócios. Os objetivos estratégicos desses programas foram 
fornecer visão e oportunidades sobre potenciais áreas de crescimento de novos negócios na nascente indústria de tecnologia (SYKES, 1990; WILSON, 2018). No início da década de 1970, 25\% das empresas listadas na Fortune 500 tinham programas de CEC ativo (FAURY; MONTEIRO, 2013; REAUME, 2003). Desde a década de 1980, iniciativas de CEC vêm sendo utilizadas para promover o empreendedorismo e para realizar as estratégias de negócios, seja de inovação, seja de busca por espaços emergentes (ARRUDA et al., 2013; BERNARDES et al., 2013). O que se argumenta é que o CEC pode ser mais barato e mais rentável do que a aquisição de uma solução tecnológica, do que a inserção no mercado ou do que o lançamento de um novo produto (WILSON, 2018).

Para Dushnistky e Lenox (2006), o CEC pode fornecer um meio eficaz de explorar o ambiente em busca de novas tecnologias para os negócios, de aumentar o poder de negociação com fornecedores e de permitir maior acesso às redes de distribuição. Segundo os autores, o CEC também fornece acesso a valiosas redes de profissionais, e isto é relevante, pois a falta de mão de obra qualificada tem se tornado um dos principais problemas para as empresas (EDELMAN INTELLIGENCE, 2018; ROMANS, 2016). Em consequência, o CEC passou a ser uma alternativa que as grandes corporações têm usado para lidar com as rápidas mudanças do mercado, buscando em empresas nascentes as soluções inovadoras (ou a promessa de) que não foram capazes de desenvolver internamente.

Os esforços em CEC são caracterizados por serem de natureza estratégica e/ou financeira. Os CECs estratégicos são direcionados para promoverem a abertura à inovação e aos conhecimentos externos (VON KROGH et al., 2012), incluindo acesso a ativos complementares que seriam caros e demorados para construir (BASU; PHELPS; KOTHA, 2011). Os CECs financeiros buscam altos retornos sobre o capital investido (FAURY; MONTEIRO, 2013). Diferentes estudos concluíram que os objetivos na dimensão estratégica se sobrepõem aos financeiros, considerando principalmente que as empresas buscam fomentar a cultura de inovação, acelerar a entrada de ideias inovadoras em seus pipelines e incorporar o conhecimento externo em seus processos internos de inovação (DUSHNITSKY; LENOX, 2006). Associado à natureza do Capital Empreendedor Corporativo estão algumas motivações que as organizações têm reportado para a busca dessa iniciativa.

\subsection{Motivações para o uso do Capital Empreendedor Corporativo}

A literatura especializada indica que a adoção do capital empreendedor corporativo é motivada por alguns fatores principais. A natureza estratégica concentra as motivações relacionadas à capacidade da empresa em gerar conhecimento e aplicá-lo para a continuidade de suas ações de inovação e alinhamento com o mercado (ARRUDA et al., 2013; BASU et al., 2016; BATTISTINI; HACKLIN; BASCHERA, 2013; CHEMMANUR; LOUTSKINA; TIAN, 2014; FAURY; MONTEIRO, 2013; ROMANS, 2016). Complementam-se as essas motivações, a interação para mudanças comportamentais apoiadas na captação de talentos, na promoção do intraempreendedorismo e na alteração da própria estrutura e da atuação das equipes internas de inovação (DIRIK, 2017; HENDERSON, 2009; RIECHE; FARIA, 2014; WADHWA; PHELPS; KOTHA, 2015).

Estão relacionadas à natureza financeira do $\mathrm{CEC}$ as ações que visam impactar na receita ou na produtividade da empresa para redução de custo ou para ganho operacional (ANOKHIN; WINCENT; OGHAZI, 2016; VON KROGH et al., 2012). Essas ações são de 
retorno de curto e médio prazo e são direcionadas pelo objetivo e pelo grau com que as operações da empresa investidora e a startup estão vinculadas (CHESBROUGH, 2002).

Por fim, as ações que permeiam as naturezas financeira e estratégica estão vinculadas às ações da empresa em identificar oportunidades que irão impactar seu desempenho frente ao mercado, seja na proteção ou efetivamente na implementação de novo produto ou mercado (ANOKHIN; PECK; WINCENT, 2016; CHEMMANUR; LOUTSKINA; TIAN, 2014; REAUME, 2003). O Quadro 1 a seguir sintetiza as motivações e as agrupa conforme a natureza do CEC (estratégica ou financeira).

\section{Quadro 1 - Quadro síntese da literatura sobre os motivos para adoção do CEC}

\begin{tabular}{|c|c|c|}
\hline Motivações & Natureza & Autores \\
\hline $\begin{array}{l}\text { Explorar inovações } \\
\text { Explorar inovações bem-sucedidas no mercado. }\end{array}$ & Estratégica & $\begin{array}{l}\text { (ROMANS, 2016); } \\
\text { (CHEMMANUR; LOUTSKINA; TIAN, } \\
\text { 2014) }\end{array}$ \\
\hline $\begin{array}{l}\text { Acesso a novas tendências } \\
\text { Acesso a tecnologias, modelos de negócios e tendências } \\
\text { vinculadas ao negócio principal da empresa e seus } \\
\text { perímetros. }\end{array}$ & Estratégica & $\begin{array}{l}\text { (ANOKHIN; PECK; WINCENT, 2016) } \\
\text { (BATTISTINI; HACKLIN; BASCHERA, } \\
\text { 2013) } \\
\text { (ANOKHIN; WINCENT; OGHAZI, 2016) }\end{array}$ \\
\hline $\begin{array}{l}\text { Desenvolvimento de parcerias } \\
\text { Identificar parcerias com startups de tecnologia em que a } \\
\text { empresa pode tornar-se cliente, fazer parceria para colocar } \\
\text { os produtos ou serviços da startup nas mãos da força de } \\
\text { vendas da empresa. }\end{array}$ & Estratégica & $\begin{array}{l}\text { (ROMANS, 2016) } \\
\text { (BATTISTINI; HACKLIN; BASCHERA, } \\
2013 \text { ) } \\
\text { (CHESBROUGH, 2002) } \\
\text { (DIRIK, 2017) }\end{array}$ \\
\hline $\begin{array}{l}\text { Desenvolvimento de intraempreendedorismo } \\
\text { Desenvolver novos conjuntos de habilidades } \\
\text { empreendedoras em executivos que podem se unir a } \\
\text { programas internos de CEC, visando fomentar o } \\
\text { intraempreendedorismo, comercializar tecnologias } \\
\text { desenvolvidas internamente e desenvolver novos negócios. }\end{array}$ & Estratégica & $\begin{array}{l}\text { (HENDERSON, 2009) } \\
\text { (BATTISTINI; HACKLIN; BASCHERA, } \\
2013 \text { ) } \\
\text { (DIRIK, 2017) }\end{array}$ \\
\hline $\begin{array}{l}\text { Desenvolvimento de imagem empreendedora } \\
\text { Criar uma imagem para o público, demonstrando que a } \\
\text { empresa é inovadora e atrativa para trabalhar, para comprar } \\
\text { ou fazer negócios com startups. }\end{array}$ & Estratégica & $\begin{array}{l}\text { (ROMANS, 2016) } \\
\text { (CHESBROUGH, 2002) }\end{array}$ \\
\hline $\begin{array}{l}\text { Captação de talentos } \\
\text { Acessar capital humano e influenciar o seu grupo de } \\
\text { recursos humanos para melhor saber que tipo de pessoas } \\
\text { contratar. }\end{array}$ & Estratégica & $\begin{array}{l}\text { (ROMANS, 2016) } \\
\text { (DIRIK, 2017) }\end{array}$ \\
\hline $\begin{array}{l}\text { Globalização da empresa } \\
\text { Estabelecer escritórios e operações em diferentes países e } \\
\text { núcleos de tecnologia, impulsionando a globalização da } \\
\text { empresa. }\end{array}$ & Estratégico & $\begin{array}{l}\text { (ROMANS, 2016) } \\
\text { (ANOKHIN; PECK; WINCENT, 2016) }\end{array}$ \\
\hline $\begin{array}{l}\text { Ampliação de } P \& D \\
\text { Substituir ou aumentar o time e o processo de } P \& D \\
\text { corporativo, considerando inovações internas e externas, } \\
\text { livrando-se de suas limitações. }\end{array}$ & Estratégica & $\begin{array}{l}\text { (WADHWA; PHELPS; KOTHA, 2015) } \\
\text { (BATTISTINI; HACKLIN; BASCHERA, } \\
\text { 2013) } \\
\text { (DIRIK, 2017) }\end{array}$ \\
\hline $\begin{array}{l}\text { Participação acionária em empresas nascentes } \\
\text { Criar oportunidades para a empresa beneficiar-se da } \\
\text { participação acionária em startups de estágio inicial e/ou } \\
\text { de crescimento, em que a empresa permitirá que essas } \\
\text { startups rapidamente se tornem empresas muito valiosas. }\end{array}$ & Financeira & $\begin{array}{l}\text { (ROMANS, 2016) } \\
\text { (WEIBLEN; CHESBROUGH, 2015) } \\
\text { (DIRIK, 2017) }\end{array}$ \\
\hline $\begin{array}{l}\text { Aumento de eficiência } \\
\text { Aumentar a eficiência da empresa, por meio da redução de } \\
\text { custos, adotando tecnologia ou utilizando soluções da } \\
\text { empresa investidas. }\end{array}$ & Financeira & $\begin{array}{l}\text { (ROMANS, 2016) } \\
\text { (VON KROGH et al., 2012) } \\
\text { (ANOKHIN; WINCENT; OGHAZI, 2016) }\end{array}$ \\
\hline Retorno financeiro & Financeira & (CHESBROUGH, 2002) \\
\hline
\end{tabular}


Altos retornos financeiros sobre o capital investido.

Tolerância ao fracasso/ risco

Permitir que as empresas concretizem seu projeto antes de interromper seu investimento, tendo maior tolerância aos riscos.

Desenvolver pipeline de oportunidades

Criar um pipeline de oportunidades de investimento por meio de programas maduros ou nascentes que permitam investimentos diretos e apresentem retorno estratégico e financeiro.

Acesso a novos mercados

Entrada em novos mercados e defesa de mercado de que já participa.

Fonte: Elaborado pelos autores.
(DIRIK, 2017)

Financeira/ (CHEMMANUR; LOUTSKINA; TIAN, Estratégica 2014)

Financeira/

(ANOKHIN; PECK; WINCENT, 2016) (BATTISTINI; HACKLIN; BASCHERA, 2013)

Financeira/ Estratégica
(ANOKHIN; PECK; WINCENT, 2016) (REAUME, 2003)

(DIRIK, 2017)

A base de conhecimento sobre as motivações para a adoção do CEC, entretanto, deriva da realidade de países com mais tradição de inovação e com mercados financeiros mais desenvolvidos. Portanto, é preciso entender se as motivações citadas nos trabalhos internacionais também estão presentes em países menos desenvolvidos economicamente, com menos tradição de inovação e com mercado financeiro não tão desenvolvido, como o Brasil.

\subsection{Capital Empreendedor Corporativo no Brasil}

O setor de Capital Empreendedor é muito recente no Brasil, foi iniciado com a experiência do BNDES em 1974, com a criação de três unidades voltadas a atividades afins, que se integraram a partir de 1982, com o nome BNDESPAR (FARIA MEIRELLES; PIMENTA JÚNIOR; REBELATTO, 2008).

De forma análoga, o CEC ainda não é uma prática amplamente adotada (ARRUDA et al., 2013). No Brasil, o CEC tem sido marcado pela interação entre os atores públicos e privados, para explorar e alavancar oportunidades de revitalização das empresas, construção de novas capacidades, renovação estratégica, retornos de investimentos, busca por inovações e aumento do desempenho competitivo (MUNDO NETO; SALTORATO, 2017; RODRIGUES; SANTOS; KUNIYOSHI, 2013).

Salusse e Campos Neto (2018) destacam que os fatores críticos de sucesso dos programas de CEC são sua consistência e seu alinhamento com a estratégia da empresa. À medida que o conceito de CEC se torna mais difundido, e que as empresas percebem valor em sua adoção, ele ganha acentuado impulso (RIECHE; FARIA, 2014).

A literatura nacional especializada em CEC tem documentado a dinâmica relacional entre as startups e as grandes empresas (MENDONÇA, 2017), e como as empresas têm desenvolvido iniciativas de empreendedorismo corporativo por meio do CEC (SALUSSE; CAMPOS NETO, 2018). Essas pesquisas também têm explorado as características e os processos utilizados no CEC (SIQUEIRA; CARVALHO; GALLUCCI NETTO, 2011) e as tendências no processo de implementação e acompanhamento de oportunidades do CEC (BERNARDES et al., 2013; FARIA MEIRELLES; PIMENTA JÚNIOR; REBELATTO, 2008; FAURY; MONTEIRO, 2013). Além disso, a pesquisa brasileira tem estudado como o CEC tem se tornado fonte de recursos para as startups e aceleradoras (SANTOS FIGUEIREDO; FIGUEIREDO; BRAGA, 2017). 
Os trabalhos sobre CEC também têm documentado que, no Brasil, as grandes empresas buscam investir em empresas menores (startups) ou no ecossistema de inovação (aceleração e incubação), com o propósito de ampliar suas capacidades de gerar novas receitas por meio da ampliação da base de clientes, da criação e reformulação de produtos ou negócios, do estímulo ao processo de inovação interna, da reformulação da cultura e da ampliação da capacidade de absorção de conhecimento (ARANTES, 2017; FAURY; MONTEIRO, 2013). Em complemento ao capital investido, as empresas com programas de CEC auxiliam os empreendedores na profissionalização da gestão do negócio, contribuindo, por exemplo, com experiência profissional e expansão da rede de contatos da empresa que recebe o investimento (FAURY; MONTEIRO, 2013).

Levantamento realizado pela HBS Angels Brazil, apoiado pela FINEP, MCTIC, ABDI e Inovativa Brasil, com empresas que possuem programas de CEC no Brasil, constatou que os principais objetivos apontados por essas iniciativas estavam vinculados aos objetivos estratégicos, à resolução de problemas, ao rejuvenescimento da cultura corporativa, à projeção da imagem da empresa (empresa inovadora) e aos retornos financeiros (ARANTES, 2017).

O Quadro 2 estabelece um comparativo entre as motivações encontradas nos trabalhos internacionais e as motivações encontradas na literatura nacional. Observa-se que os itens de natureza Financeira são comuns. No entanto, os itens vinculados à natureza estratégica, relacionados ao posicionamento da empresa frente ao mercado internacional e a tolerância ao fracasso/risco não são apontados na literatura brasileira. Isso pode ser decorrente do fato das empresas brasileiras ainda concentrarem boa parte de seus esforços no curto prazo, para resolução de problemas, projeção de imagem e retorno financeiro, dentro das necessidades especificas do cenário brasileiro (ARANTES, 2017; FAURY; MONTEIRO, 2013; LUIZA et al., 2018). Entretanto, as divergências encontradas podem ser decorrentes também do fato de as motivações registradas pela literatura nacional não serem derivadas de pesquisas com a finalidade específica de entender o propósito do uso do CEC no Brasil. Desse modo, a literatura nacional tem apenas referenciado o que a literatura estrangeira indica como motivações, carecendo de pesquisas no país que explorem de forma sistematizada o porquê do uso do CEC no Brasil.

Quadro 2 - Comparativo de motivações para uso do CEC

\begin{tabular}{|c|c|c|c|c|}
\hline Motivações & Natureza & $\begin{array}{c}\text { Literatura } \\
\text { Internacional }\end{array}$ & $\begin{array}{l}\text { Literatura } \\
\text { Brasileira }\end{array}$ & Referências Brasileiras \\
\hline Explorar inovações & Estratégica & $\mathrm{X}$ & $\mathrm{X}$ & $\begin{array}{l}\text { (BERNARDES et al., 2013; } \\
\text { FARIA MEIRELLES; } \\
\text { PIMENTA JÚNIOR; } \\
\text { REBELATTO, 2008; FAURY; } \\
\text { MONTEIRO, 2013) } \\
\text { (MUNDO NETO; } \\
\text { SALTORATO, 2017) }\end{array}$ \\
\hline Acesso a novas tendências & Estratégica & $\mathrm{X}$ & $\mathrm{X}$ & (ALUMNI BRASIL, 2017) \\
\hline Desenvolvimento de Parcerias & Estratégica & $\mathrm{X}$ & $\mathrm{X}$ & $\begin{array}{l}\text { (LUIZA et al., 2018) } \\
\text { (ALUMNI BRASIL, 2017) }\end{array}$ \\
\hline $\begin{array}{l}\text { Desenvolver Pipeline de } \\
\text { Oportunidades }\end{array}$ & $\begin{array}{l}\text { Financeira/ } \\
\text { Estratégica }\end{array}$ & $\mathrm{X}$ & $\mathrm{X}$ & $\begin{array}{l}\text { (BERNARDES et al., 2013; } \\
\text { FARIA MEIRELLES; } \\
\text { PIMENTA JÚNIOR; }\end{array}$ \\
\hline
\end{tabular}




\begin{tabular}{|c|c|c|c|c|}
\hline & & & & $\begin{array}{l}\text { REBELATTO, 2008; FAURY; } \\
\text { MONTEIRO, 2013) }\end{array}$ \\
\hline $\begin{array}{l}\text { Participação Acionária em } \\
\text { empresas nascentes }\end{array}$ & Financeira & $\mathrm{X}$ & $X$ & $\begin{array}{l}\text { (SANTOS FIGUEIREDO; } \\
\text { FIGUEIREDO; BRAGA, 2017) }\end{array}$ \\
\hline $\begin{array}{l}\text { Desenvolvimento de } \\
\text { intraempreendedorismo }\end{array}$ & Estratégica & $\mathrm{X}$ & $X$ & $\begin{array}{l}\text { (SALUSSE; CAMPOS NETO, } \\
\text { 2018) } \\
\text { (ARANTES, 2017; FAURY; } \\
\text { MONTEIRO, 2013) } \\
\text { (ARANTES, 2017; FAURY; } \\
\text { MONTEIRO, 2013) }\end{array}$ \\
\hline $\begin{array}{l}\text { Desenvolvimento imagem } \\
\text { empreendedora }\end{array}$ & Estratégica & $\mathrm{X}$ & $X$ & (ARANTES, 2017) \\
\hline Captação de Talentos & Estratégica & $\mathrm{X}$ & $X$ & (ALUMNI BRASIL, 2017) \\
\hline Aumento de Eficiência & Financeira & $\mathrm{X}$ & $X$ & $\begin{array}{l}\text { (MUNDO NETO; } \\
\text { SALTORATO, 2017) }\end{array}$ \\
\hline Acesso a novos mercados & $\begin{array}{l}\text { Financeira/ } \\
\text { Estratégica }\end{array}$ & $\mathrm{X}$ & $X$ & $\begin{array}{l}\text { (ARANTES, 2017; FAURY; } \\
\text { MONTEIRO, 2013) } \\
\text { (ALUMNI BRASIL, 2017) }\end{array}$ \\
\hline Globalização da Empresa & Estratégica & $\mathrm{X}$ & & \\
\hline Ampliação de $P \& D$ & Estratégica & $\mathrm{X}$ & $X$ & $\begin{array}{l}\text { (MUNDO NETO; } \\
\text { SALTORATO, 2017) } \\
\text { (ARANTES, 2017; FAURY; } \\
\text { MONTEIRO, 2013) }\end{array}$ \\
\hline Retorno Financeiro & Financeira & $X$ & $X$ & $\begin{array}{l}\text { (ARANTES, 2017; FAURY; } \\
\text { MONTEIRO, 2013) } \\
\text { (MUNDO NETO; } \\
\text { SALTORATO, 2017) }\end{array}$ \\
\hline $\begin{array}{l}\text { Tolerância ao Fracasso / } \\
\text { Risco }\end{array}$ & $\begin{array}{l}\text { Financeira/ } \\
\text { Estratégica }\end{array}$ & $X$ & & \\
\hline
\end{tabular}

Fonte: Elaborado pelos autores.

\section{Método de Pesquisa}

Para entender o porquê das empresas estarem buscando estruturar iniciativas de Capital Empreendedor Corporativo (CEC), a presente pesquisa utilizou uma abordagem qualitativa. A pesquisa qualitativa foi considerada mais apropriada, tendo em vista a preocupação com os aspectos da realidade da empresa, direcionando-se na clareza e interpretação das relações sociais e no universo de significados, motivos, aspirações, crenças, valores e atitudes que envolvem sua capacidade de inovação (GERHARDT; SILVEIRA, 2009). Assim, o estudo profundo e exaustivo de um ou poucos objetos, neste caso as empresas do agronegócio brasileiro, permitiu seu amplo e detalhado conhecimento (GIL, 2002), encarando o delineamento mais adequado para a investigação de um fenômeno contemporâneo, dentro de seu contexto real, em que os limites entre o fenômeno e o contexto não são claramente percebidos (YIN, 2015). Portanto, a metodologia de estudo de caso foi aplicada com o propósito de identificar, de forma sistemática, as motivações para as empresas do setor do agronegócio brasileiro usarem o modelo de CEC.

\subsection{Contexto}

O setor agrícola brasileiro tem experimentado rápida evolução de modo a atender as necessidades globais de produzir mais alimentos em área cultivável cada vez menor e sem degradar o meio ambiente (DUTIA, 2014). Os avanços tecnológicos têm contribuído para o 
aumento dos índices de produtividade agropecuária. Um número menor de pessoas no campo tem produzido mais (ARAÚJO, 2007). E isso se deve, dentre outras coisas, ao fato de que as tecnologias que aumentam a produção e a eficiência agrícola ganharam força nos últimos anos (WHOLEY, 2018). A modernização da agricultura brasileira tem promovido, nos últimos 40 anos, profunda reestruturação dos espaços produtivos do campo, a partir da incorporação de novas tecnologias nas etapas do trabalho agrícola, possibilitando o aperfeiçoamento das atividades econômicas e a especialização produtiva das regiões (SANTOS, 2014). Portanto, o setor tem experimentado rápidas mudanças em seus processos produtivos (AGROPENSA, 2014).

O setor tem atraído um número crescente de fundos de investimentos, grandes companhias de tecnologia e empreendedores (MELO, 2016). Com isso, o setor agrícola tem presenciado um movimento que poderá se tornar uma das grandes ondas tecnológicas no futuro próximo (WALKER et al., 2016). O 'Radar AgTech Brasil 2019 - Mapeamento das Startups do Setor Agro Brasileiro', co-realizado pela Embrapa, SP Ventures e Homo Ludens, identificou 1125 startups, ilustrando a complexidade e diversidade nos diversos segmentos da cadeia (DIAS; JARDIM; SAKUDA, 2019).

Diante desse cenário, os modelos de investimento para interação com o ecossistema de inovação para o agronegócio se tornam cada vez mais necessários, motivo pelo qual o CEC tem sido buscado pelas empresas como uma alternativa para apoiar os desafios do setor (AGROPENSA, 2014; WALKER et al., 2016).

\subsection{Casos}

Para responder à pergunta de pesquisa, foram estudados os casos de duas empresas. A seleção dos casos obedeceu aos seguintes critérios: empresas que tenham relação com agronegócio brasileiro e que, nos últimos cinco anos, tenham utilizado iniciativas de CEC como mecanismo para interações com atores externos para os processos de inovação.

A solicitação de entrevista foi enviada para oito empresas que estavam aderentes aos critérios acima, conforme pesquisas prévias na internet e em documentos do setor. No entanto, algumas não deram continuidade à iniciativa e outras não estavam disponíveis. Portanto, a amostra foi por conveniência e ficou restrita às empresas 'Máquinas Agrícolas Jacto' e 'TOTVS', as únicas empresas com o perfil desejado disponíveis para participar desta pesquisa.

De forma a ampliar o entendimento do setor e da interação das grandes empresas com empresas menores num contexto de CEC, além dos dois casos, foram realizadas entrevistas com dois especialistas em CEC. Um dos especialistas é sócio de uma empresa dedicada a prestar assessoria na estruturação de CECs. O especialista é empreendedor na área de tecnologia há 25 anos, tendo fundado sua primeira empresa aos 19 anos. Atualmente, é consultor em Fusões e Aquisições para empresas em estágio de crescimento e em 'Corporate Venture' para grandes empresas, além de ser investidor anjo em novos negócios (startups). Ele é um dos fundadores da 'Anjos do Brasil', entidade criada para fomentar o investimentoanjo para apoio ao empreendedorismo de inovação brasileiro. O segundo especialista é sócio de uma gestora de recursos, responsável pelo fundo de mais de R 100 milhões, cuja carteira possui diversos investimentos em empresas de tecnologia voltadas à cadeia agropecuária. 
O estudo dos casos foi feito a partir de análise documental e entrevistas com os gestores das empresas selecionadas, sendo que as entrevistas foram realizadas por meio de roteiro semiestruturado, com vistas a, se necessário, aprofundar assuntos específicos sobre a realidade do fenômeno de interesse.

A primeira fase ocorreu por meio de exploração de dados secundários, relatórios internos, conteúdos de website e outros documentos disponíveis (YIN, 2015). A triangulação foi feita a partir da comparação de dados diferentes, com o propósito de validar os achados (GRÄBNER; EISENHARDT, 2007). Nesses documentos, tomou-se o cuidado de não utilizar o discurso da empresa, mas selecionar as informações relevantes que pudessem auxiliar e completar as entrevistas realizadas. Os dados secundários foram solicitados para os responsáveis da empresa ou coletados no próprio site institucional das empresas. $\mathrm{Na}$ segunda fase, foram realizadas, presencialmente ou via videoconferência, as entrevistas com os gestores e com os especialistas.

\section{Análise e Discussão}

Os resultados da pesquisa confirmam os principais aspectos encontrados na literatura: as empresas observaram no CEC, frente a outros investimentos, uma alternativa para atender à necessidade de crescimento, competitividade e representatividade de sua base de clientes no mercado (ARRUDA et al., 2013; BERNARDES et al., 2013; CHESBROUGH, 2002); para ampliar a busca de novas tecnologias para os negócios (DUSHNITSKY; LENOX, 2006); para acesso a novas competências e profissionais de mercado (EDELMAN INTELLIGENCE, 2018; ROMANS, 2016).

No Brasil, revela-se forte demanda por parte das empresas para que os projetos de inovação, apoiados pelo CEC, apresentem retorno financeiro para suas efetivas implementações. Isso revela a baixa tolerância ao risco por parte das empresas para os investimentos a partir do modelo de CEC. As empresas que usam CEC visam ampliar seus processos de inovação e aderência às oportunidades de mercado, mas com ações que não almejam a maior possibilidade de risco. Na percepção dos especialistas, esse fato é retratado em iniciativas de curto prazo, em que as empresas procuram resultados práticos e rápidos nos programas que apoiam. Segundo um entrevistado, existe uma pressão muito forte das empresas por inovação, "as grandes empresas estão sendo pressionadas por novos modelos de inovação disruptivos, mas internamente precisam mudar sua cultura para serem mais tolerantes a riscos".

Esta pesquisa também revelou que as motivações e a estrutura das empresas para uso do CEC ainda estão em formação. Segundo um entrevistado, no CEC, "gradativamente as empresas criam estruturas e começam a criar uma cultura de dinamismo que renova e mantém a empresa atualizada para o mercado". Para os especialistas, o ganho estratégico do CEC dá uma visão do futuro do mercado, das oportunidades, da organização e do planejamento, que podem gerar vantagem competitiva frente aos potenciais concorrentes.

Diante das constatações mencionadas acima, é esperado que o uso do CEC ainda tenha ampliação dos seus objetivos (i.e., motivações), considerando que os investimentos das empresas devem ocorrer cada vez mais para aumentar a capacitação dos times gestores, e o alinhamento de suas estratégias para uma visão de continuidade e expansão de mercados por meio de processos de inovação, com iniciativas que extrapolam o curto prazo. 
Conforme Quadro 3, observa-se que, apesar de o CEC ser um mecanismo que pode dar maior flexibilidade às empresas quanto à tolerância ao fracasso, o cenário encontrado no recorte realizado neste estudo não corrobora tal possibilidade.

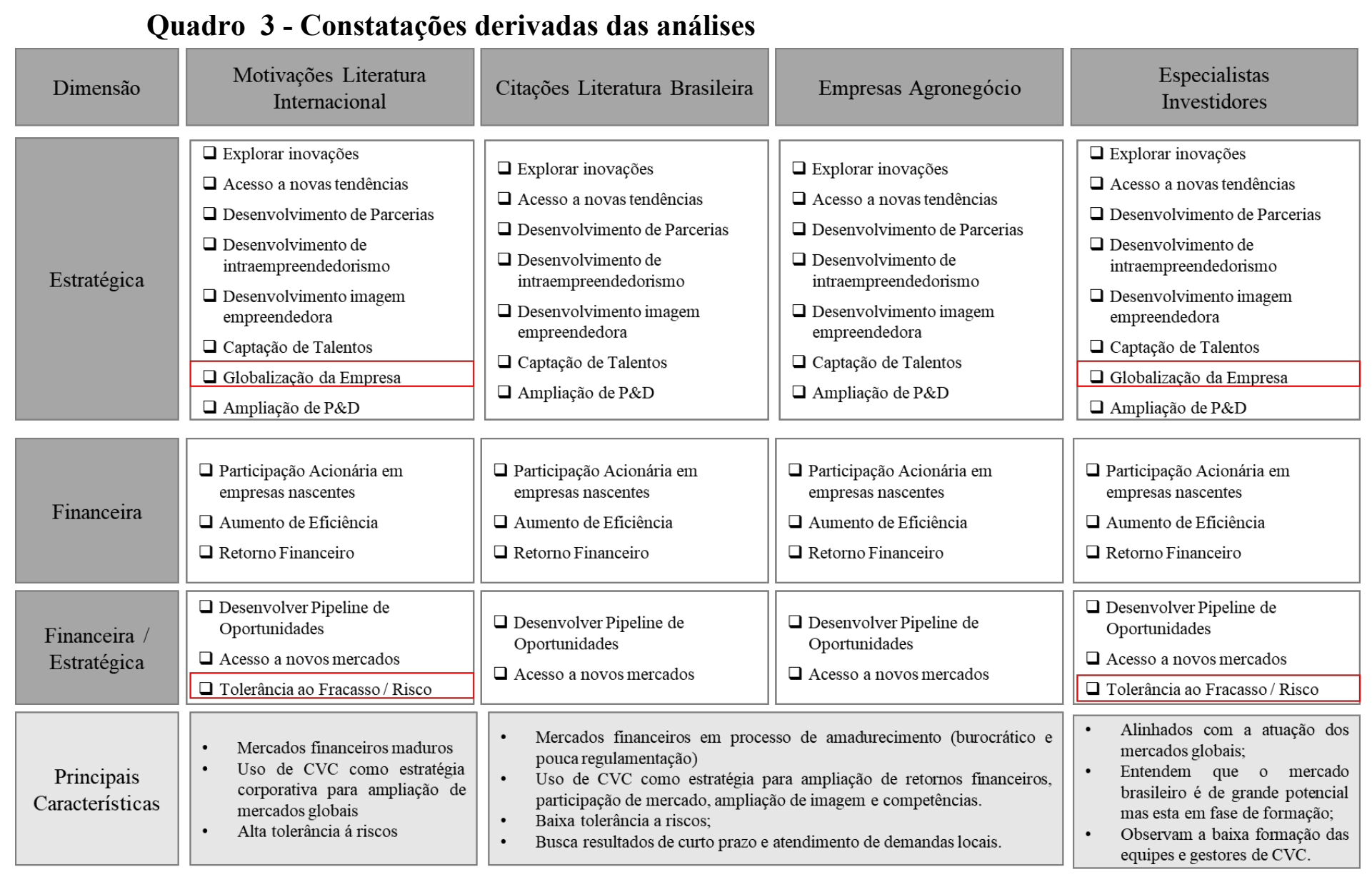

Fonte: Elaborado pelos autores.

Os itens sinalizados em vermelho são as motivações não abordadas nas publicações brasileiras e não foram mencionadas pelos gestores das empresas pesquisadas. Dessa forma, é possível considerar um fluxo para interação do processo de inovação com as iniciativas de CEC, antes de efetivamente as empresas interagirem com os atores externos para execução e seja ampliado o processo de validação dos objetivos com entidades de fomento (investidores, órgãos regulatórios, Universidades e Entidades Internacionais), de forma a consolidar seus objetivos e capacitar as equipes gestoras de CEC. Diferente da forma como hoje são realizadas ações em que a Equipe Gestora já interage com aceleradores e startups para o desenvolvimento de soluções e inovações.

\section{Considerações Finais}

O objetivo deste trabalho foi explorar as motivações que têm levado as grandes empresas a utilizar o CEC como forma de investimento, para a ampliar sua interação com 
atores externos e, assim, aumentar sua capacidade de inovação. Por meio de revisão de publicações da área, buscou-se entender se as motivações citadas nas pesquisas internacionais, para uso de Capital Empreendedor Corporativa, são comuns ao cenário brasileiro, com recorte específico no agronegócio.

Como resultado, as características dos investimentos no Brasil demonstram que a principal função do CEC está voltada para retorno financeiro, com iniciativas de curto prazo. Mesmo que as ações tenham apelos estratégicos, os indicadores de avaliação e direcionamento focam nos retornos de ganho financeiro. Em complemento, as evidências observadas demonstram uma aversão ao risco de curto, médio e longo prazos. Essas condições são suportadas pelas questões legais quanto a poucas garantias para o investidor, o momento econômico e a incerteza da aderência da inovação frente as necessidades do mercado. Os investimentos são avaliados, considerando principalmente a resolução de problemas ou uma lacuna muito clara de potencial ganho de mercado.

Em suplementação aos casos estudados foi possível constatar que: em ambientes de negócios, cujo mercado financeiro e o ecossistema de inovação ainda estão em amadurecimento, o modelo de CEC não é usado para apoiar iniciativas de elevados riscos e incertezas; e em ambientes de negócios, cujo mercado financeiro e o ecossistema de inovação ainda estão em amadurecimento, as estruturas internas para realizar o CEC são definidas para rápidas ações com o ecossistema de inovação, mas com poucas interações com entidades, como investidores, órgãos regulatórios, Universidades e entidades internacionais.

Respondendo à pergunta de pesquisa de porque as empresas do agronegócio estão investindo em CEC, conclui-se que é uma forma de estar próximo do ecossistema de inovação e de ampliar a capacidade de inovar. Por outro lado, essas ações são direcionadas fortemente para retornos financeiros, mas com pouco apetite a risco, o que compromete a visão da empresa em atender mercados internacionais. Em linha com esse cenário, as dimensões dos investimentos recaem sobre os indicadores de retorno financeiro e participação no mercado.

Um outro fator que emergiu, a partir dos casos estudados, foi a falta de clareza das empresas ao iniciarem os programas de CEC. $\mathrm{Na}$ visão dos especialistas, ainda falta conhecimento e preparo das empresas que, muitas vezes, iniciam programas sem uma estratégia definida e buscam proximidade com o ecossistema de inovação sem propósitos definidos, o que posteriormente impacta na continuidade dos programas.

Observa-se processo de amadurecimento nas iniciativas das empresas brasileiras para uso do CEC. Ainda há fortes oportunidades de amadurecimento dos programas para explorar iniciativas que possam ampliar seu mercado de atuação, analisando o potencial das soluções para atendimento de mercados internacionais e flexibilização de seus processos para maior tolerância a riscos.

Dessa forma, entre as contribuições deste estudo de caso no âmbito acadêmico, destacase a importância de contemplar-se esse setor tão significativo para o país nas pesquisas e nos estudos relativos CEC, como fonte de investimento para processo de inovação, ampliando as análises das motivações e estruturas que levam as empresas a fazerem uso desse mecanismo. Acrescenta-se o trabalho a respeito da Corporate Venture Capital, principalmente no tocante ao impacto dessa relação interorganizacional no desenvolvimento de novas tecnologias. Quanto às contribuições profissionais, este estudo revela a importância de as empresas 
investirem na capacitação dos colaboradores que atuam com CEC dentro de suas estruturas, bem como uma maior interação com entidades de fomento para consolidação de estratégias e ampliação de suas fronteiras para entrega de produtos e serviços a outros países.

Como limitações do estudo, tem-se a análise específica de um setor e a quantidade de empresas entrevistadas, com análise transversal, sem recorte longitudinal. Apesar da relevância das empresas no setor e do reconhecimento de suas ações inovadoras, a ampliação dos casos analisados traria maior abrangência às análises.

Como estudos futuros, sugerem-se explorar outros segmentos para validar as motivações de uso do CEC, bem como validar o quanto a baixa aceitação de riscos pode impactar na ampliação dos programas de CEC no Brasil e nos processos de inovação desses segmentos.

\section{Referências:}

AGROPENSA. Visão 2014-2034 O Futuro do Desenvolvimento Tecnológico da Agricultura Brasileira. Embrapa, p. 194, 2014.

AGTHENTIC. A Guide to Startup Resources for Agriculture and Food Technology Innovation Bridging the gaps between innovation, investment, and business. AgFounder, p. 1-15, 2016.

ANOKHIN, S.; PECK, S.; WINCENT, J. Corporate venture capital: The role of governance factors. Journal of Business Research, v. 69, n. 11, p. 4744-4749, 2016.

ANOKHIN, S.; WINCENT, J.; OGHAZI, P. Strategic effects of corporate venture capital investments. Journal of Business Venturing Insights, v. 5, n. 7, p. 63-69, 1 jun. 2016.

ARANTES, M. V. Status de Corporate Venture no Brasil: Como grandes empresas Estão Se Relacionando com o ecossistema empreendedor. HBS Alumni Angels Of Brazil, p. 44, 2017.

ARAÚJO, M. J. Fundamentos de Agronegócio. 2a Edição ed. São Paulo: Atlas SA, 2007. ARRUDA, C; COZZI, A.; SOUZA, G.; PENIDO, E. Towards an understanding of corporate venturing practices in Brazil. Venture Capital, v. 15, n. 2, p. 135-149, 2013.

BATTISTINI, B.; HACKLIN, F.; BASCHERA, P. The State of Corporate Venturing: Insights from a Global Study. Research-Technology Management, v. 56, n. 1, p. 31-39, 2013.

BEECHAM RESEARCH. Towards Smart Farming Agriculture Embracing the IOT Vision. Beecham Research, 2014.

BERMAN, D.; WAI, S. The Next Food Frontier: How AgTech Can Save The World. Disponível em: <https://techcrunch.com/2015/09/07/the-next-food-frontier-how-agtechcan-save-the-world/>. Acesso em: 18 set. 2018.

BERNARDES, R. C.; VARELA, C.A.; CONSONI, F. L.; SACRAMENTO, E. S. Ensaio sobre as virtudes do capital empreendedor corporativo para projetos de alta tecnologia no setor agrícola: a trajetória inovadora da Alellyx Applied Genomics e da CanaVialis. Revista de Administração, v. 48, n. 2, p. 327-340, 2013.

BIRKINSHAW, J.; BOUQUET, C.; BARSOUX, J. L. Top 10 Lessons on the New Business of Innovation (among which Chesbrough: The Era of OI). MIT Sloan Management Review, v. 52, n. 1, p. 1-80, 2011.

CHEMMANUR, T. J.; LOUTSKINA, E.; TIAN, X. Corporate Venture Capital, Value 
Creation, and Innovation. The Review of Financial Studies, v. 27, n. 8, p. 2434-2473, 2014. CHESBROUGH, H. Open Innovation: Where We've Been and Where We're Going. Research-Technology Management, v. 55, n. 4, p. 20-27, 2012.

CHESBROUGH, H.; CROWTHER, A. K. Beyond high tech: early adopters of open innovation in other industries. R\&D Management, v. 36, n. 3, p. 229-236, 2006.

CHESBROUGH, H. W. Making Sense of Corporate Venture Capital. Harvard Business Review, v. 80, n. 3, p. 90-99, 2002.

COSTA, P. R. DA; PORTO, G. S. Proposição de uma tipologia para a internacionalização de P\&D interno e externo nas multinacionais brasileiras. Revista de Administração, v. 48, n. 1, p. 145, 2013.

CRESWELL, J. W. Projeto de Pesquisa. 2a Edição ed. Porto Alegre: Bookman, 2007. CRIATEC. Desmistificando o Capital Empreendedor. Rio de Janeiro: Criatec, 2009.

CUMMING, D.; FLEMING, G.; SUCHARD, J.-A. Venture capitalist value-added activities, fundraising and drawdowns. Journal of Banking \& Finance, v. 29, n. 2, p. 295-331, 1 fev. 2005.

DIAS, C. N.; JARDIM, F.; SAKUDA, L. O. (Orgs.) Radar AgTech Brasil 2019: Mapeamento das Startups do Setor Agro Brasileiro. Embrapa, SP Ventures e Homo Ludens: Brasília e São Paulo, 2019. Disponível em: <www.radaragtech.com.br>. Acesso em: 30 set. 2019.

DIRIK, I. A Manual for Corporate Venture Capital. Boston Consulting Group, 2017.

DUSHNITSKY, G.; LENOX, M. J. When does corporate venture capital investment create firm value? Journal of Business Venturing, v. 21, n. 6, p. 753-772, 2006.

DUTIA, S. AgTech: Challenges and Opportunities for Sustainable Growth. Available at SSRN 2431316, v. 9, n. April, 2014.

ECOAGRO. O Agronegócio no Brasil - Ecoagro. Ecoagro Group, 2013.

EDELMAN INTELLIGENCE. GE Global Innovation Barometer 2018 Full Report. GE Global Innovation Barometer, 2018.

EMBRAPA, S. VI Plano Diretor da Embrapa: 2014-2034. EMBRAPA, p. 1-28, 2015.

FACCHINETTI, C. Produção e consumo de produtos orgânicos crescem $20 \%$ ao ano no país. Disponível em: <https:/www.anufoodbrazil.com.br/2018/07/13/producao-e-consumode-produtos-organicos-crescem-20-ao-ano-no-pais/>. Acesso em: 18 set. 2018.

FARIA MEIRELLES, J. L.; PIMENTA JÚNIOR, T. P.; REBELATTO, D. A. DO N. Venture capital e private equity no Brasil: alternativa de financiamento para empresas de base tecnológica. Gestão \& Produção (UFSCAR. Impresso), v. 15, n. 1, p. 11-21, 2008.

FAURY, T. P.; CARVALHO, M. M. Corporate venture capital: geração e acompanhamento de oportunidades de investimento em empresas inovadoras. Produção, v. 56, n. 4, p. 735$750,2013$.

GASSMANN, O.; ENKEL, E.; CHESBROUGH, H. The future of open innovation. R\&D Management, v. 40, n. 3, p. 213-221, 2010.

GERHARDT, T. E.; SILVEIRA, D. T. Métodos de pesquisa. 1a Edição ed. Porto Alegre: SEAD/UFRGS, 2009.

GIL, A. C. Como Elaborar Projetos de Pesquisa. 4a Edição ed. São Paulo: Atlas, Editora, 2002.

GRÄBNER, M. E.; EISENHARDT, K. M. Theory building from cases: opportunities and 
challenges. Academy of Management Journal, v. 50, n. 1, p. 25-32, 2007.

HENDERSON, J. The role of Corporate Venture Capital funds in financing biotechnology and healthcare: differing approaches and performance consequences. International Journal of Technoentrepreneurship, v. 2, n. 1, p. 29, 2009.

KARVINEN-NIINIKOSKI, S. Social Work Supervision: Contributing to Innovative Knowledge Production and Open Expertise. Social work, critical reflection and the learning organization. Routledge, n. 2003, p. 23-39, 2004.

LEE, S. M.; KIM, T.; JANG, S. H. Inter-organizational knowledge transfer through corporate venture capital investment. Management Decision, v. 53, n. 7, p. 1601-1618, 2015.

LOPES, A. P. V. B. V.; CARVALHO, M. M. DE. Evolução da literatura de inovação em relações de cooperação: um estudo bibliométrico num período de vinte anos. Gestão \& Produção, v. 19, n. 1, p. 203-217, 2012.

LUIZA, A. et al. Mecanismos de Geração de Empreendimentos: As Aceleradoras de StartUps no Brasil. GEINTEC, v. 8, n. 1, p. 4187-4199, 2018.

MAIA, C. et al. Fatores da cultura organizacional que condicionam ou limitam o processo de inovação. Revista Capital Científico - Eletrônica, v. 12, n. 3, p. 150-163, 2014.

MELO, C. O que é AgTech e por que o Brasil pode liderar essa nova onda tecnológica. StartAgro, 2016.

MENDONÇA, H. L. O Impacto das Startups no Setor de Energia. FGV Energia, 2017.

MOHAMED, A.; SCHWIENBACHER, A. Voluntary disclosure of corporate venture capital investments. Journal of Banking \& Finance, v. 68, n. 1, p. 69-83, 1 jul. 2016.

MUNDO NETO, M.; SALTORATO, P. Atores, Ações e Dominação Cultural na Construção da Indústria de Capital Empreendedor no Brasil. Estudos de Sociologia, v. 22, n. 43, p. 217 234, 2017.

REAUME, A. Is Corporate Venture Capital a Prescription for Success in the Pharmaceutical Industry? The Journal of Private Equity, v. 6, n. 4, p. 77-87, 2003.

RIECHE, F. C.; FARIA, L. R. B. DE. O Corporate Venturing como alternativa de apoio à Inovação - Motivações e Benefícios. Revista do BNDES, n. 41, p. 379-414, 2014.

RODRIGUES, F. Z.; SANTOS, S. A. DOS; KUNIYOSHI, M. S. Modelos de Corporate Venturing de Campbell: estudos de múltiplos casos sobre seus usos em empresas atuantes no Brasil. Revista Pensamento \& Realidade, v. 28, n. 2, p. 46-61, abr. 2013.

ROMANS, A. Masters of Corporate Venture Capital: Noth Charleston, South Carolina: Createspace Independent Publishing Platform, 2016.

SALUSSE, M. A. Y.; CAMPOS NETO, N. M. DE. A Hora e a vez do Empreendedorismo Corporativo. GVExecutivo, v. 17, n. 3, p. 22-23, 2018.

SANTOS FIGUEIREDO, L. H. DOS; FIGUEIREDO, D.; BRAGA, S. DE A. M. Impactos do Programa de Aceleração SEED do Estado de Minas Gerais. Anprotec, v. 1, n. 1, p. 20, 2017.

SANTOS, H. F. Modernização da Agricultura e Relação Campo-Cidade: Uma Análise a partir do Agronegócio Cafeeiro no Município de Alfenas - MG. Caminhos de Geografia, v. 15, n. 51, p. 154-171, 2014.

SIQUEIRA, E. M. R.; CARVALHO, A. G. DE; GALLUCCI NETTO, H. Determinantes do sucesso dos investimentos de private equity e venture capital no Brasil. Revista Brasileira de Finanças, v. 9, n. 1, p. 190-208, 2011. 
SYKES, H. B. Corporate venture capital: Strategies for success. Journal of Business Venturing, v. 5, n. 1, p. 37-47, 1990.

TEECE, D. J. Business Models, Business Strategy and Innovation. Long Range Planning, v. 43, n. 2-3, p. 172-194, 2010.

THEES, A. Corporate venture in Brazil gains steam as giants amp up startup investments | TechCrunch. Tech Crunch, 2016.

TIWARI, R.; HERSTATT, C. Open Global Innovation Networks as Enablers of Frugal Innovation: Propositions Based on Evidence from India. Hamburg University of Technology, Technology and Innovation Management, Working Paper, v. 72, p. 35, 2012.

VODAPHONE; ACCENTURE; OXFAM. Connected Agriculture. Accenture, p. 42, 2011. VON KROGH, G.; BATTISTINI, B; PACHIDOU, F.; BASCHERA, P.. The changing face of corporate venturing in biotechnology. Nature Biotechnology, v. 30, n. 10, p. 911-915, 2012.

WADHWA, A.; PHELPS, C.; KOTHA, S. Corporate venture capital portfolios and firm innovation. Journal of Business Venturing, v. 31, n. 1, p. 95-112, 2015.

WALKER, D. et al. Lessons from the Front Lines of the Agtech Revolution. Boston Consulting Group, 2016.

WAYRA BRASIL. Por que as AgTechs podem revolucionar nosso futuro. Disponível em: $\quad<$ https://medium.com/wayrabrasil/por-que-as-agtechs-podem-revolucionar-nossofuturo-3d97c996dbcd >. Acesso em: 18 set. 2018.

WEIBLEN, T.; CHESBROUGH, H. W. Engaging with Startups to Enhance Corporate Innovation. California Management Review, v. 57, n. 2, p. 66-90, fev. 2015.

WHOLEY, M. Agtech and the Connected Farm CB Insights, 2018.

WILSON, F. History of Corporate Venture Capital. CB Insights, 2018.

YIN, R. K. Estudo de Caso: Planejamento e Métodos. 5a Edição ed. Porto Alegre: Bookman editora, 2015. 